Received: 2018.05.03

Accepted: 2018.07.26

Published: 2018.12 .01

\title{
Long Noncoding RNA Plasmacytoma Variant Translocation 1 (PVT1) Promotes Colon Cancer Progression via Endogenous Sponging miR-26b
}

Authors' Contribution: Study Design A Data Collection B Statistical Analysis C Data Interpretation D Manuscript Preparation E Literature Search F Funds Collection G

\author{
ABF 1 Rui Zhang \\ BCD 1 Jibin Li \\ $A B C 1$ Xiaofei Yan \\ BD 1 Keer Jin \\ ABD 1 Wenya Li \\ DE 1 Xin Liu \\ CF 1 Jianfeng Zhao \\ CD 1 Wen Shang \\ BDFG 2 Yefu Liu
}

1 Department of Colorectal Surgery, Cancer Hospital of China Medical University, Liaoning Cancer Hospital and Institute, Shenyang, Liaoning, P.R. China

2 Department of Hepatopancreatobiliary Surgery, Cancer Hospital of China Medical University, Liaoning Cancer Hospital and Institute, Shenyang, Liaoning, P.R. China
Corresponding Author:

Source of support: Center for Colorectal Cancer (No. 2015225005), Liaoning BaiQianWan Talents Program (No. 2017-B44)

Background:

Material/Methods:

Results:

Conclusions:

\section{MeSH Keywords:}

Full-text PDF:
Recently, long noncoding RNAs (IncRNAs) have received wide attention in the area of tumor progression. Dysregulation of IncRNAs has been shown to participated in colon cancer, a known malignant tumor. This study aimed to identify the way IncRNA PVT1 affects the progression of colon cancer.

Both human colon cancer tissues and 30 paired adjacent normal tissue samples, as well as the colon cancer cells, were collected. Then quantitative real-time (qRT-PCR) was performed to detect the expression of IncRNA PVT1 and miR-26b. Furthermore, the role of PVT1 was determined by function assays such as cell proliferation assay, invasion assay, and wound healing assay. The mechanism was studied using western blot assay and luciferase assay.

We demonstrate that the expression of PVT1 was significantly higher in tumor tissue compared with the adjacent normal tissue with a lower expression of miR-26b. Moreover, PVT1 promoted tumor growth, migration, and invasion in vitro. In addition, further experiments revealed that miR-26b was a direct target of PVT1 and could inhibit cell migration, invasion, and proliferation in colon cancer.

Our results suggest that PVT1 could promote metastasis and proliferation of colon cancer via endogenous sponging and inhibiting the expression of miR-26b, which may highlight the significance of IncRNA PVT1 in colon cancer tumorigenesis.

\section{Cell Proliferation • Colonic Neoplasms • RNA, Long Noncoding}

https://www.medscimonit.com/abstract/index/idArt/910955

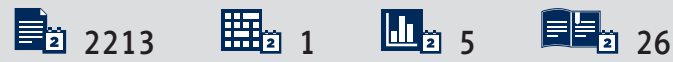




\section{Background}

Colorectal cancer (CRC) is the fourth most common diagnosed cancer and ranks as the second leading cause of cancer related death worldwide [1]. Though the quality of life for CRC patients have significantly improved by current treatment strategies, the 5-year survival rate is still only $31 \%$ in China [2], principally due to the late diagnosis time and recurrence/metastasis of tumor cells [3]. Therefore, exploring the key molecular mechanisms involved in CC progression would have a significant impact for CRC therapy. Long noncoding RNAs (IncRNAs) are defined as transcripts comprising more than 200 nucleotides in length without encoding proteins and previously believed to be transcriptional "noise" [4]. Increasing evidence revealed that IncRNAs have significant potential application prospect in the diagnosis, treatment, and prognosis in malignant tumor, including colon cancer (CC) [5]. For instance, IncRNA breast cancer anti-estrogen resistance 4 (BCAR4) was reported highly expressed in CC and by energizing of Wnt/ $\beta$-catenin signaling pathway facilitated CC cell proliferation and migration [6]. LncTCF7 could promote metastasis and invasion of CRC cell through Wnt/ $\beta$-catenin signaling pathway [7]. LncRNA SUMO1P3 expedites the malignant behaviors of $C C$ through regulating the levels of cyclin D1, vimentin, and VEGFA [8].

The novel IncRNA plasmacytoma variant translocation 1 (PVT1) is 1716 nucleotides in length locates near Myc at human chromosome8q24 [9] and in a variety of carcinoma studies the oncogenic roles of PVT1 have already been reported. Additionally, PVT1 was discovered associated with tumor size, advanced histological grades, metastases, poor prognosis, and cisplatin resistance of CRC patients, it is a significant regulator in tumorigenesis and cisplatin resistance of CRC [10]. However, the specific molecular mechanism of PVT1 involved in the progression of CC remains largely unclear.

The assembling of evidence established that through the microRNAs (miRNAs) response elements (MREs), IncRNA may act as molecular sponges for a miRNA, thereby depresses the expression of the respective miRNAs' targets, which is proposed as competing endogenous RNA (ceRNA) hypothesis. Emerging data indicated that this regulatory action plays important roles in CRC development [11]. For example, long intergenic nonprotein-coding RNA 1567 (LINC01567) which is upregulated in CC stem cells can act as a "sponge" against miRNA-93 to regulate the proliferation and tumorigenesis [12]. LnCRNA MALAT1 may mediate HMGB1 by sponging miR-129-5p, then induce CC development [13]. Upregulated HNF1A-AS1 can promote the metastatic progression of CC serving as a competing endogenous RNA to modulate miRNA-34a expression, subsequently with repression of miR-34a/SIRT1/p53 feedback loop and activation of canonical Wnt signaling pathway [14].

MiR-26b, which is a significant miRNA family member, was shown to participate in diverse human neoplasms biological functions. For instance, miR-26b could regulate invasion and migration of lung cancer cells through targeting hENT1 depending on RhoA/ROCK-1 pathway [15]. By suppressing COX-2 expression, miR-26b could inhibit glioma proliferation in vitro and in vivo [16]. In addition, miR-26b, which is downregulated by irradiation, could suppress autophagy in breast cancer cells by targeting DRAM1 mRNA [17]. However, the function of miR-26b in CC and the relevance between PVT1 and miR-26b remains unknown.

In our current study, we found that miR-26b is decreased in CC cells and it is a target of IncRNA PVT1. Through sponging miR-26b, IncRNA PVT1 promotes the migration, invasion and proliferation of colon cells. Our findings may be a valuable reference into the roles of IncRNA in CC progression.

\section{Material and Methods}

\section{Human CC tissue specimens and cell lines}

Thirty pairs of CC tissues were collected. CC cell lines SW480, NCM460, and HCT116 were procured from Shenyang Medical University. The cells were maintained at $37^{\circ} \mathrm{C}$ and $5 \% \mathrm{CO} 2$ in RPMI-1640 medium supplemented with $10 \%$ fetal bovine serum (FBS) and $1 \%$ PS (100 mg/mL streptomycin sulfate and $100 \mathrm{U} / \mathrm{mL}$ penicillin sodium).

\section{Plasmid construction}

All primers used are illustrated in Table 1 and all constructs were validated by DNA sequencing.

\section{RNA extraction and quantitative real-time PCR (qRT-PCR)}

Total RNA from CC tissues and cell lines were isolated by TRIzol reagent (Invitrogen, Carlsbad, USA) according to the manufacturer's instructions. NanoDrop-2000 spectrophotometer was

Table 1. qPCR primer sequences.

\begin{tabular}{ccc}
\hline Gene name & Forward & Reverse \\
\hline PVT1 & GCGAGCAAAGATGTGCCCC & GACCGCCAACATCCTTTCCG \\
\hline miR-26b & CCGGGACCCAGTTCAAGTAA & CCCCGAGCCAAGTAATGGAG \\
\hline
\end{tabular}




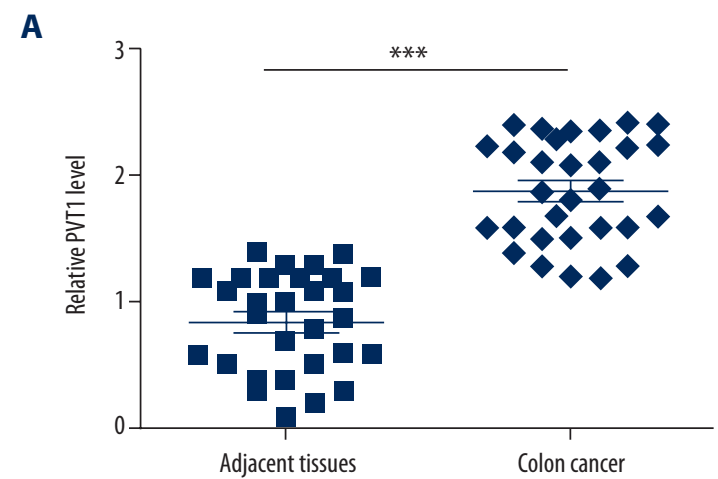

C

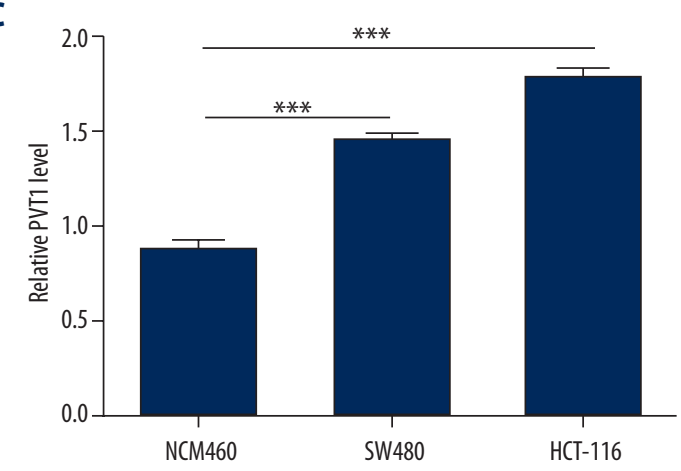

B

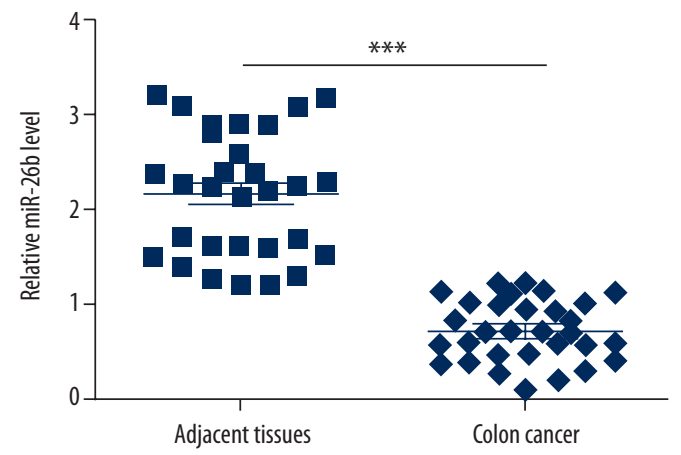

D

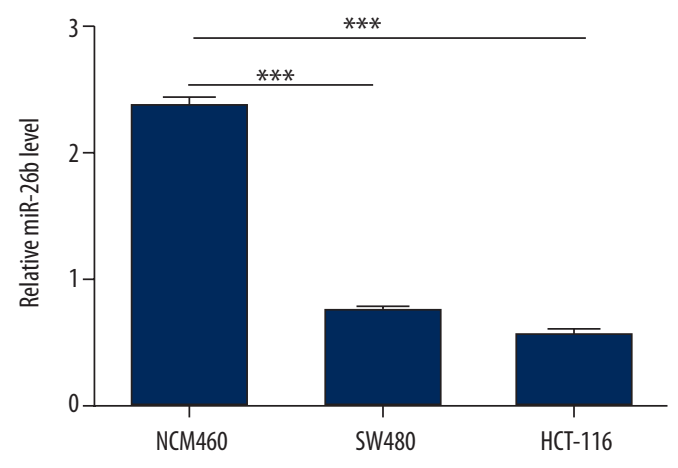

$\mathbf{E}$

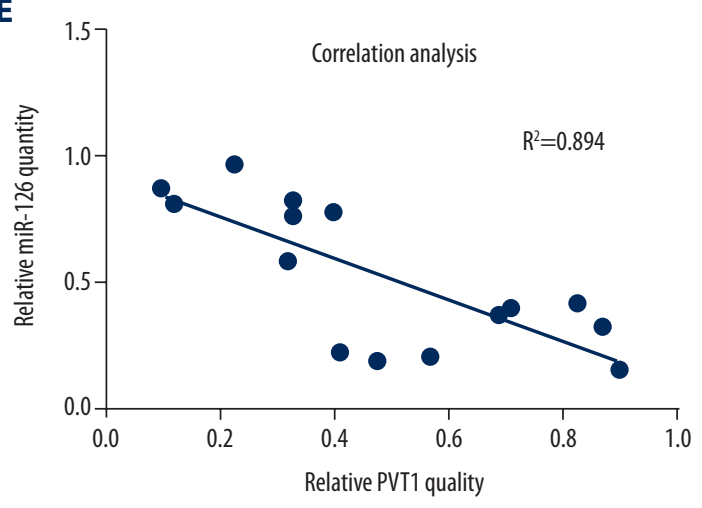

Figure 1. Differential expression of IncRNA PVT1 and miR-26b in CC tissues and cells. (A) The relative expression level of PVT1 in 30 pairs of CC tissues and adjacent non-tumor tissues; $\beta$-actin was used for normalization. (B) The mRNA levels of miR-26b were detected with qRT-PCR in 30 cases of CC. (C, D) The relative mRNA expression levels of PVT1 (C) and miR-26b (D) in NCM460, SW480, and HCT116 cell lines were assessed by qRT-PCR. (E) Pearson's correlation analysis between miR-26b and PVT1 expression in CC tissues. The data are shown as the mean \pm SD of 3 independent experiments. $\left({ }^{* *} P<0.001\right)$. CC - colon cancer; IncRNA - long noncoding RNA.

used to confirm the quantity and quality of the extracted RNA. RNA was reversely transcribed by M-MLV (Promega, Madison, WI, USA), and then Premix ExTaq II Kit (Takara) was used to perform qPCR. The relative fold changes of candidate genes in the transcripts were analyzed with $2^{-\Delta \Delta C t}$ method. The specific primers are shown in Table 1.

\section{Luciferase reporter assay}

The wild-type or mutant PCA1 binding miR-26b was subcloned into a pGL3-basic vector (Promega) to form the reporter vectors named as pGL3-PVT1-WT (PVT1-WT) and pGL3-PVT1MUT (PVT1-MT). Then, HCT116 cells were cultured in 24 well plates and co-transfected constructed luciferase plasmids 
A

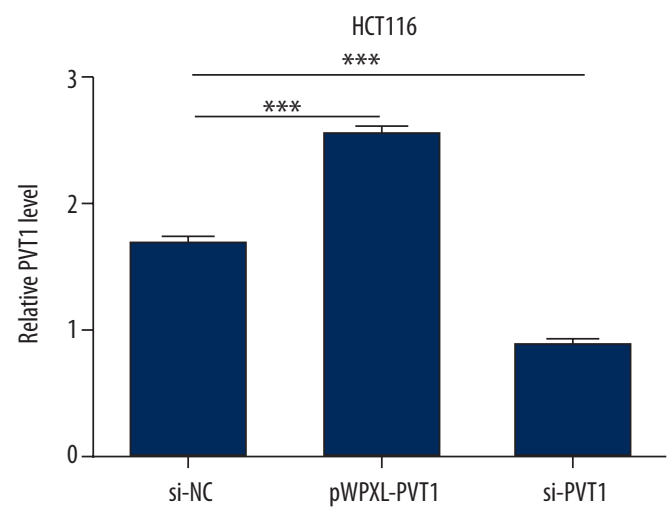

B

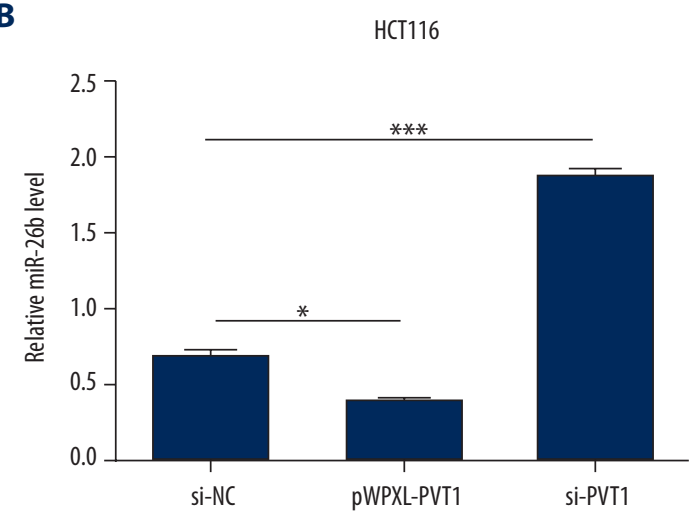

Figure 2. LncRNA PVT1 downregulates the expression of miR-26b. (A) The mRNA level of PVT1 in HCT116 cell was measured using qRT-PCR following transfection with pWPXL-PVT1 and si-PVT1 plasmids, respectively. (B) Forty-eight hours after transfection, the mRNA level of PVT1 in HCT116 cell transfected with PVT1 mimics and PVT1 inhibitors plasmids are determined with qRTPCR. The data are shown as the mean \pm SD of 2 independent experiments. ( $\left.{ }^{* *} P<0.001\right)$. LncRNA - long noncoding RNA.

together with the internal control vector containing Renilla luciferase, pRL-TK (Promega), and miR-26b or miR-control using Lipofectamine 2000 transfection reagent (Invitrogen). After 48-hour post-transfection, HCT116 cells were lysed and the Dual-Luciferase Reporter Assay System (Promega) were used for monitoring the luciferase activity.

\section{Cell proliferation assay}

Cell proliferation in vitro was detected by MTT assay. Briefly, after transfection, the density of CC cell lines was 2000 cells/well in 96-well plates and all groups were respectively incubated for 24 hours and 48 hours. MTT was added into each well ( $5 \mathrm{mg} / \mathrm{mL}$, Sigma, USA), and culture was maintained at $37^{\circ} \mathrm{C}$ for 4 to 6 hours. Samples were measured at $570 \mathrm{~nm}$ using the MTT cell proliferation kit (Cayman Chemical).

\section{Wound healing assay}

We incubated cells in 6-well plates with normal cell growth medium. When the culture reaches $85 \%$, we scratched the cell layer using $20 \mu \mathrm{L}$ sterile pipette tip and then washed it in culture medium, then cultured for 48 hours with the $1 \%$ FBS culture medium. Because cell proliferation may affect cells migrated, to the analysis of the wound, the cells were incubated at $37^{\circ} \mathrm{C}$ for 1 hour with mitomycin $\mathrm{C}(10 \mu \mathrm{g} / \mathrm{mL})$. We used a microscope to obtain images at different time points ( 0,48 hours).

\section{Cell invasion assays}

Transwell chamber inserts with an $8 \mu \mathrm{m}$ pore, with Matrigel, were used to analyze the invasion ability of CC cells. Then $2 \times 10^{4} \mathrm{HCT} 116$ cells were seeded into the upper compartment of each insert, which was coated with $40 \mu \mathrm{L}$ Matrigel $(2 \mathrm{mg} / \mathrm{mL})$.
While the bottom of the insert was incubated with $500 \mu \mathrm{L}$ RPMI 1640 with $20 \%$ FBS. Then, the chambers were maintained for 24 hours at $37^{\circ} \mathrm{C}$ in $5 \% \mathrm{CO} 2$. Afterwards, the cells were fixed, stained, and measured through the pores by calculating under a 20x microscope (Zeiss, Germany).

\section{Statistical analysis}

Statistical analysis was performed using Prism 5.0 (GraphPad Prism) software. Experimental results are presented as means \pm standard deviation. The comparisons between the groups were conducted with Student's t-test or ANOVA with multiple comparison with $P$ value less than 0.05 considered statistically significant.

\section{Results}

\section{LncRNA PVT1 was highly expressed while miR-26b was downregulated in CC tissues and cell lines}

To explore the expression profiles of PVT1 and miR-26b in CC, qRT-PCR analysis was performed in CC tissues and adjacent normal tissues. The results showed that PVT1 was remarkably overexpressed in the cancer samples compared with the non-cancerous tissues (Figure 1A). Conversely, miR-26b was downregulated significantly in the cancer samples (Figure 1B). Furthermore, the same observations were obtained in NCM460 cells, SW480 cells, and HCT116 cells (Figure 1C, 1D). As expected, the correlation analysis showed that the expression of miR-26b had a negatively correlation with PVT1 (Figure 1E). Collectively, these results showed that PVT1 was expressed highly in CC tissues and cell lines with a lower expression of miR-26b. 


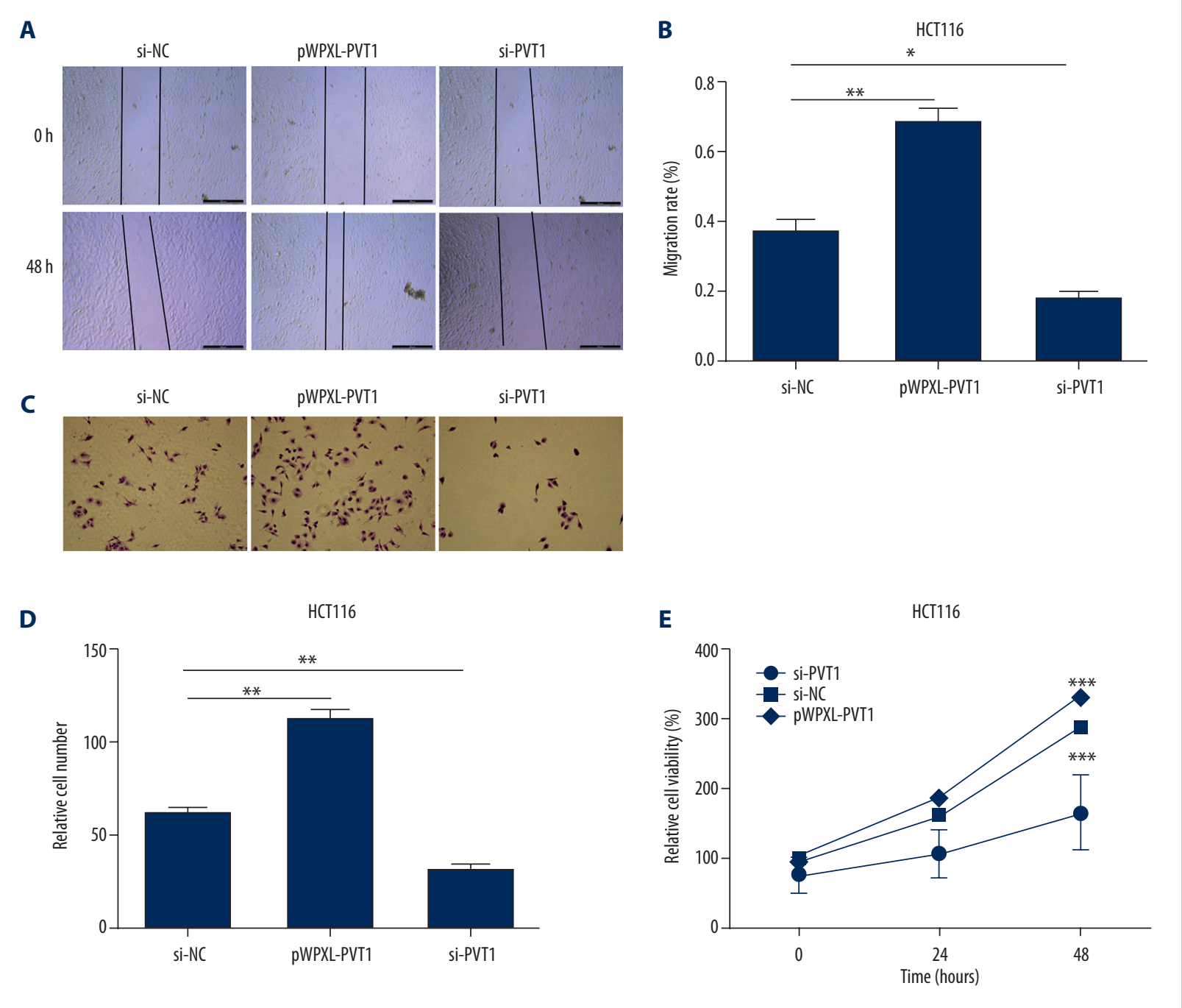

Figure 3. LncRNA PVT1 promotes colon cell migration, invasion, and proliferation. (A, B) Wound healing assays were performed with cells transfected with either pWPXL-PVT1 or si-PVT1 to reveal the relative mobility. (C, D) Twenty-four hours posttransfection, cell invasion was evaluated in HCT116 cells using a Transwell system with $8 \mu \mathrm{m}$ pores in polycarbonate membranes. Representative views are shown. All photomicrographs were taken at 200x magnification. (E) MTT assay was performed to determine the cell proliferation transfected with pWPXL-PVT1 and si-PVT1 in HCT116 cells. The data are shown as the mean $\pm \mathrm{SD}$ of 3 independent experiments. $\left({ }^{*} P<0.5 ;{ }^{* *} P<0.01 ;{ }^{* * *} P<0.001\right)$.

\section{LncRNA PVT1 regulated the expression of miR-26b}

To explore the relationship between PVT1 and miR-26b in CC, IncRNA PVT1 mimics and inhibitors were constructed and the effectiveness was verified using qRT-PCR (Figure 2A). As shown in Figure $2 \mathrm{~B}$, the mRNA levels of miR-26b were marked decreased after PVT1 overexpressed, and remarkably increased when PVT1 was knocked down in HCT116 cells. These results suggest that PVT1 inhibited the expression of miR-26b in HCT116 cells.

\section{LncRNA PVT1 promoted colon cell migration, invasion, and proliferation}

Since the expression levels of PVT1 were markedly increased in CC tissues and cells, we speculated that it might be involved in CC progression. To estimate the function of PVT1 on the malignant behaviors of human CC, wound healing assay was conducted. As shown in Figure 3A and 3B, when PVT1 was overexpressed, the migration capacity of HCT116 cell was significantly increased, while PVT1 knockdown decreased cell migration ability of HCT116 cells. Next, we performed Transwell assays to assess the invasion abilities of HCT116 cells. The results showed that pWPXL-PVT1 enhanced CC cell invasion ability, but si-PVT1 


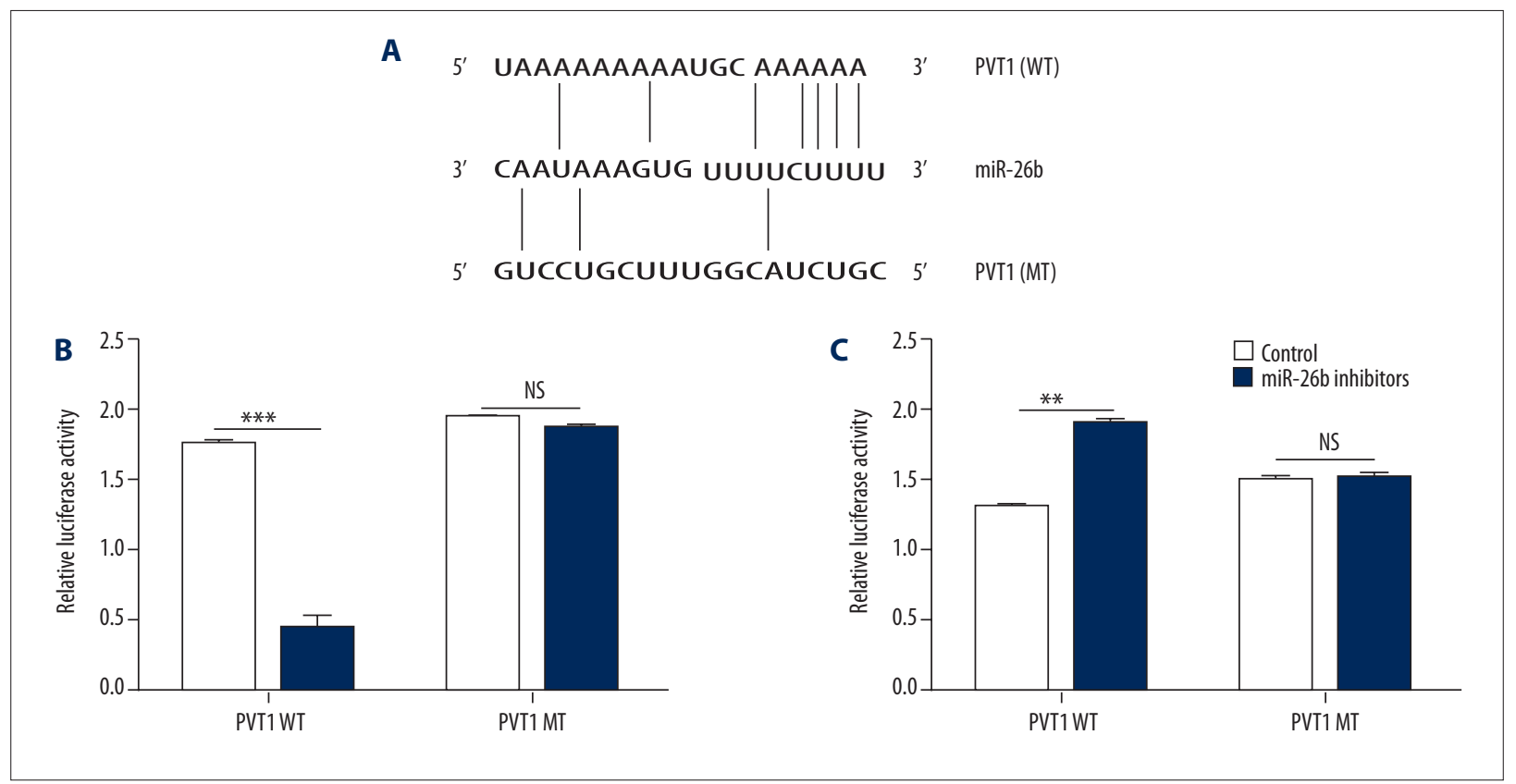

Figure 4. LncRNA PVT1 directly interacted with miR-26b. (A) Sequence alignment and schematic diagram of miR-26b with the wildtype (top) and mutated (bottom) 3'UTR of PVT1. (B, C) The luciferase activity of the reporter vectors was detected in HCT116 cells after 48 hours of co-transfection wide type or mutant PVT1-3'UTR with miR-26b respectively; pRL-TK was used for normalization. The data are shown as the mean \pm SD of 3 independent experiments. ${ }^{* *} P<0.01 ;{ }^{* * *} P<0.001$; ns - not significant). LncRNA - long noncoding RNA.

inhibited the invasion ability of CC cells, which was consistent with the results of wound healing assays (Figure 3C, 3D). Then, an MTT assay was performed to determine whether PVT1 influenced cell viability. Compared with the control, HCT116 cells transected with pWPXL-PVT1 showed a significant increase in cell viability. Conversely, HCT116 cells transected with si-PVT1 exhibited decreased cell viability (Figure 3E).

\section{LncRNA PVT1 directly interacted with miR-26b}

Accumulating evidence has suggested that IncRNAs can act as a competing endogenous RNA by binding to specific miRNAs, we conducted dual-luciferase reporter assay to confirm whether PVT1 plays its role in such a way. We subcloned the pGL3-promoter vector containing wide-type (WT) or mutant (WT) miR-26b putative binding sites in PVT1 (Figure 4A). The result of dualluciferase assays suggested that the luciferase activities were significantly decreased after we co-transfected cells with mimics PVT1-WT and miR-26b but not the PVT1-MT (Figure 4B, 4C).

\section{MiR-26b inhibited cell migration, invasion, and proliferation in CC}

To evaluate the impact of miR-26b on the progression of CC, wound healing assays were performed. The migration of HCT116 cells were remarkably reduced by the upregulation of miR-26b. And we observed the opposite result when the expression of miR-26b was suppressed (Figure 5A, 5B). As for Transwell assays, the results showed that the invasion abilities of HCT116 cells were markedly enhanced after overexpression of miR-26b, and the opposite observation was obtained when miR-26b knockdown (Figure 5C, 5D). Furthermore, the cell proliferation of HCT116 cells were monitored using MTT assays. As shown in Figure 5E, the cell viability of HCT116 cells were significantly inhibited after transected with miR-26b mimics, while miR-26b inhibitor caused an increase. Those results indicated that miR-26b inhibited cell migration, invasion, and proliferation in CC.

\section{Discussion}

LncRNAs could communicate with other RNA transcripts through miRNA response elements according to the ceRNA hypothesis as the letters of a novel RNA language [18]. Many studies have reported that interactions between IncRNAs and miRNAs play important roles in cancer progression [1]. Thus, a better understanding of the interaction between IncRNA and miRNA would be important for CC. Our study demonstrated that PVT1 could suppress the expression of miR-26b through directly interacting with it. Furthermore, PVT1 regulated migration, invasion, and proliferation of CC cells via sponging miR-26b, which provided new evidence of the existence of ceRNA regulatory network in CC cells. 


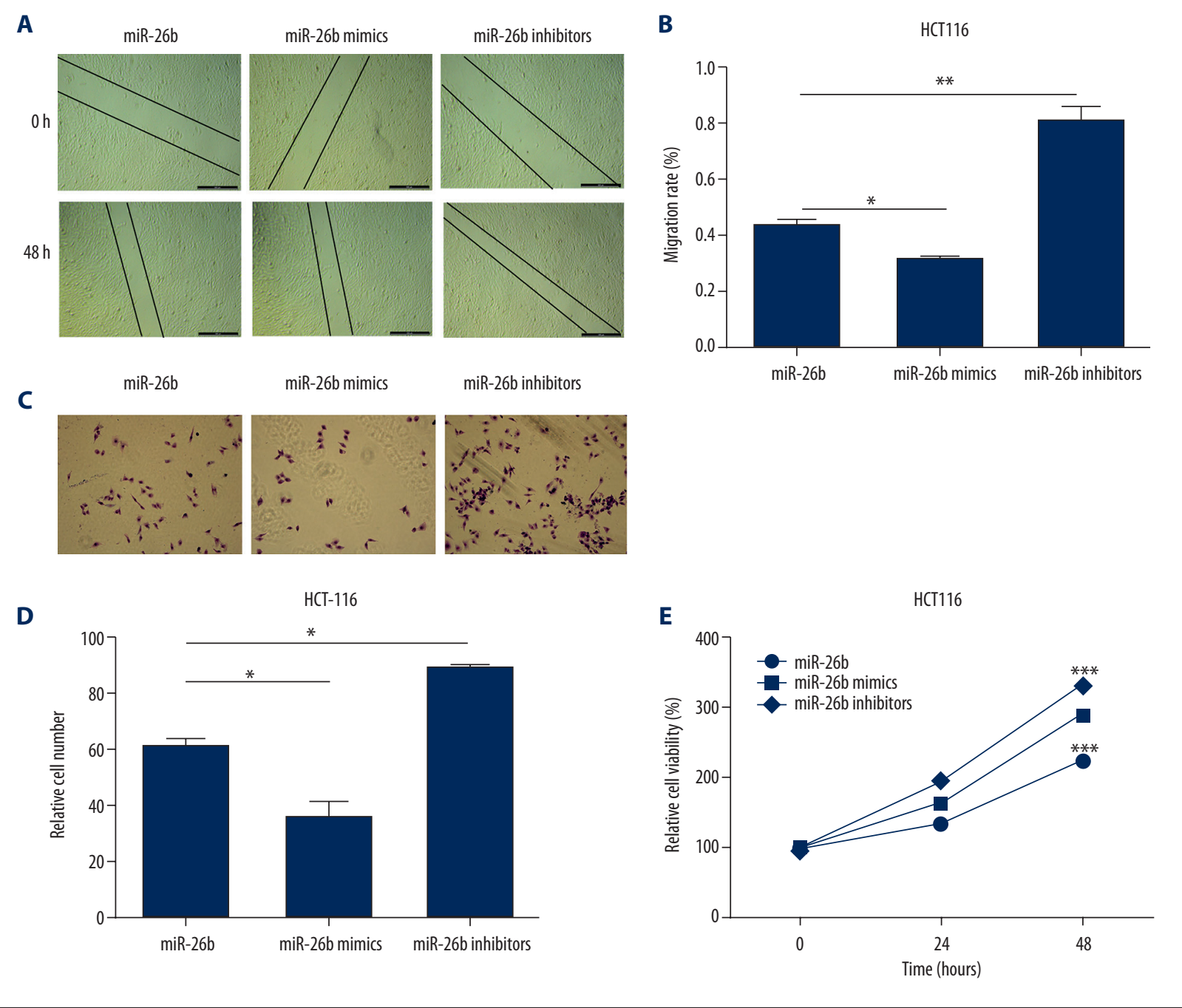

Figure 5. MiR-26b inhibits cell migration, invasion and proliferation in CC. (A, B) The effects on cell migration in HCT116 cells after transfection with miR-26b mimics and inhibitors. (C, D) Transwell assays were performed to estimate the cell invasion of HCT116 cells when transfected with corresponding plasmids. (E) Cell proliferation was investigated using MTT assay. The data are shown as the mean \pm SD of 3 independent experiments. $\left({ }^{*} P<0.5 ;{ }^{* *} P<0.01 ;{ }^{* * *} P<0.001\right)$.

Accumulated evidence has shown that PVT1 is upregulated and functions as an oncogenic IncRNA in multiple types of tumors. Xue et al. reported that PVT1 regulates the malignant behaviors of human glioma cells by targeting miR-190a-5p and miR-488-3p [20]. Chang et al. demonstrated that PVT1 can promote EMT by upregulation of Twist1, a transcription factor associated with EMT via acting as a sponge for miRNA-186-5p in prostate cancer [21]. Furthermore, PVT1 showed higher expression in nasopharyngeal carcinoma, it could predict poor prognosis, and it could induce radio-resistance by regulating DNA repair and cell apoptosis [22]. In this study, we demonstrated that PVT1 was significantly upregulated in human CC tissues and CC cell lines and that PVT1 contributed to the malignant behavior of CC cells. Specifically, PVT1 overexpression significantly enhanced the migration, invasion, and proliferation, whereas PVT1 knockdown had the opposite function. Thus, PVT1 may act as an oncogene in CC, consistent with the previous researches $[23,24]$.

The mechanism by which PVT1 regulates migration, invasion, and proliferation in CC cells remains largely undefined. To explore these mechanisms, we focused on the miR-26b, which has been shown to be remarkable dysregulation in varies of cancers, such as esophageal squamous cancer [25] and gastric cancer [26]. Furthermore, diverse downstream genes of miR-26b have been identified. Li et al. demonstrated that miR-26b could facilitate apoptosis and inhibit proliferation/invasion of neuroglioma cells via downregulating $\mathrm{Bcl}-2$ expression and potentiating caspase- 3 activity (effects of miRNA-26b on proliferation and invasion of glioma cells and related mechanisms). 
MiR-26b could also suppress esophageal cancer cell proliferation by inhibition of MYCBP, subsequently downregulating c-MYC pathway (miR-26a and miR-26b inhibit esophageal squamous cancer cell proliferation through suppression of c-MYC pathway). In addition, miR-26b might be implicated in CRC progression via direct targeting FUT4 (tumor-suppressive miR-26a and miR-26b inhibit cell aggressiveness by regulating FUT4 in colorectal cancer). Our study demonstrated that miR-26b was downregulated and inhibited cell migration, invasion, and proliferation in CC tissues and cell lines. In addition, PVT1 was evidenced to regulate the expression of miR-26b through directly interacted with it. But by which targets of miR-26b did PVT exert its function needs further research.

\section{References:}

1, Siegel RL, Miller KD, Jemal A: Cancer statistics, 2018. Cancer J Clin, 2018; 68(1): 7-30

2. Jiang Z, Li Y, Han G et al: [Association of serum albumin level with clinicopathologic features and prognosis in colon cancer]. Zhonghua Wei Chang Wai Ke Za Zhi, 2016; 19(1): 80-83 [in Chinese]

3. Fedewa SA, Flanders WD, Jemal A et al: Racial and Ethnic disparities in interval colorectal cancer incidence. Ann Intern Med, 2018; 168(1): 80-87

4. Kunej T, Obsteter J, Pogacar Z et al: The decalog of long non-coding RNA involvement in cancer diagnosis and monitoring. Crit Rev Clin Lab Sci, 2014 51(6): 344-57

5. Yarmishyn AA, Kurochkin IV: Long noncoding RNAs: A potential novel class of cancer biomarkers. Front Genet, 2015; 6: 145-52

6. Ouyang $\mathrm{S}$, Zheng $\mathrm{X}$, Zhou $\mathrm{X}$ et al: LncRNA BCAR4 promotes colon cancer progression via activating Wnt/beta-catenin signaling. Oncotarget, 2017; 8(54): 92815-26

7. Li T, Zhu J, Wang X et al: Long non-coding RNA IncTCF7 activates the Wnt/ beta-catenin pathway to promote metastasis and invasion in colorectal cancer. Oncol Lett, 2017; 14(6): 7384-90

8. Zhang LM, Wang P, Liu XM, Zhang YJ: LncRNA SUMO1P3 drives colon cancer growth, metastasis and angiogenesis. Am J Transl Res, 2017; 9(12): 5461-72

9. Chinen $\mathrm{Y}$, Sakamoto N, Nagoshi $\mathrm{H}$ et al: $8 \mathrm{q} 24$ amplified segments involve novel fusion genes between NSMCE2 and long noncoding RNAs in acute myelogenous leukemia. J Hematol Oncol, 2014; 7: 68-75

10. Ping G, Xiong W, Zhang L et al: Silencing long noncoding RNA PVT1 inhibits tumorigenesis and cisplatin resistance of colorectal cancer. Am J Transl Res, 2018; 10(1): 138-49

11. Shuwen H, Qing Z, Yan Z, Xi Y: Competitive endogenous RNA in colorectal cancer: A systematic review. Gene, 2018; 645: 157-62

12. $\mathrm{Yu} X, \mathrm{Mi}$ L, Dong J, Zou J: Long intergenic non-protein-coding RNA 1567 (LINC01567) acts as a "sponge" against microRNA-93 in regulating the proliferation and tumorigenesis of human colon cancer stem cells. BMC Cancer, 2017; 17(1): 716-22

13. Jie $\mathrm{Y}$, Zhao H: LnCRNA MALAT1 induces colon cancer development by regulating miR-129-5p/HMGB1 axis. J Cell Physiol, 2017; 11(2): 138-48

\section{Conclusions}

Our research indicated that PVT1 was upregulated in CC tissues and cell line with a significant dysregulation of miR-26b. Furthermore, PVT1 enhanced cell migration, invasion, and proliferation through negatively regulating miR-26b in CC, clarifying the molecular mechanism of the oncogenic role of PVT1 in CC, which allowed us to better understand the pathogenesis and development of CC and provided a IncRNA-directed target for this deadly disease.

14. Fang C, Qiu S, Sun F et al: Long non-coding RNA HNF1A-AS1 mediated repression of miR-34a/SIRT1/p53 feedback loop promotes the metastatic progression of colon cancer by functioning as a competing endogenous RNA. Cancer Lett, 2017; 410: 50-62

15. Gao Y, Yang F: [MiR-26b regulates invasion and migration of lung cancer cells through targeting hENT1 depending on RhoA/ROCK-1 pathway]. Zhong Nan Da Xue Xue Bao Yi Xue Ban, 2017; 42(7): 755-61 [in Chinese]

16. Chen ZG, Zheng CY, Cai WQ et al: MiR-26b mimic inhibits glioma proliferation in vitro and in vivo suppressing COX-2 expression. Oncol Res, 2017; 410: $50-70$

17. Meng C, Liu Y, Shen $Y$ et al: MicroRNA-26b suppresses autophagy in breast cancer cells by targeting DRAM1 mRNA, and is downregulated by irradiation. Oncol Lett, 2018; 15(2): 1435-40

18. Salmena L, Poliseno L, Tay Y et al: A ceRNA hypothesis: The Rosetta Stone of a hidden RNA language. Cell, 2011; 146(3): 353-58

19. Wu Q, Meng WY, Jie Y, Zhao H: LncRNA MALAT1 induces colon cancer development by regulating miR-129-5p/HMGB1 axis. J Cell Physiol, 2018; 233(9): 6750-57

20. Xue W, Chen J, Liu X et al: PVT1 regulates the malignant behaviors of human glioma cells by targeting miR-190a-5p and miR-488-3p. Biochim Biophys Acta, 2018; 1864(5 Pt A): 1783-94

21. Chang Z, Cui J, Song Y: Long noncoding RNA PVT1 promotes EMT via mediating microRNA-186 targeting of Twist1 in prostate cancer. Gene, 2018; 654: $36-42$

22. He $Y$, Jing $Y$, Wei $F$ et al: Long non-coding RNA PVT1 predicts poor prognosis and induces radioresistance by regulating DNA repair and cell apoptosis in nasopharyngeal carcinoma. Cell Death Dis, 2018; 9(2): 235-41

23. Yu X, Zhao J, He Y: Long non-coding RNA PVT1 functions as an oncogene in human colon cancer through miR-30d-5p/RUNX2 axis. J BUON, 2018; 23(1): 48-54

24. Ping $G$, Xiong $W$, Zhang $L$ et al: Silencing long noncoding RNA PVT1 inhibits tumorigenesis and cisplatin resistance of colorectal cancer. Am J Transl Res, 2018; 10(1): 138-49

25. Li J, Liang Y, Lv H et al: miR-26a and miR-26b inhibit esophageal squamous cancer cell proliferation through suppression of c-MYC pathway. Gene, 2017; 625: 1-9

26. Tsai MM, Huang HW, Wang CS et al: MicroRNA-26b inhibits tumor metastasis by targeting the KPNA2/c-jun pathway in human gastric cancer Oncotarget, 2016; 7(26): 39511-26 